\title{
Plasma glucose in screening for diabetes and pre-diabetes: how much is too much? Analysis of fasting plasma glucose and oral glucose tolerance test in Sri Lankans
}

\author{
G. W. Katulanda ${ }^{1}$, P. Katulanda ${ }^{2,3,4}$, C. Dematapitiya ${ }^{2}$, H. A. Dissanayake $2^{*}$ (D) S. Wijeratne ${ }^{5}$, M. H. R. Sheriff ${ }^{2}$ and
}

D. R. Matthews ${ }^{4}$

\begin{abstract}
Background: Fasting plasma glucose (FPG) is the most commonly used screening tool for diabetes in Sri Lanka. Cut-off values from American Diabetes Association recommendations are adopted in the absence of local data. We aimed to establish FPG cut offs for Sri Lankans to screen for diabetes and pre-diabetes.

Methods: Data on FPG and diabetes/pre-diabetes status were obtained from Sri Lanka Diabetes and Cardiovascular Study (SLDCS), a community based island wide observational study conducted in 2005-6. Sensitivity specificity and area under the ROC curve were calculated for different FPG values.

Results: Study included 4014 community dwelling people after excluding people already on treatment for diabetes or pre-diabetes. Mean age was 45.3 ( \pm 15 ) years and $60.4 \%$ were females. FPG cut off of $5.3 \mathrm{mmol} / \mathrm{L}$ showed better sensitivity and specificity than $5.6 \mathrm{mmol} / \mathrm{L}$ in detecting diabetes (87.8\% and $84.4 \%$ Vs $80.8 \%$ and $92.1 \%)$ and prediabetes (54.7\% and $87.0 \%$ Vs $43.8 \%$ and $94.2 \%)$
\end{abstract}

Conclusions: A lower FPG cut off of $5.3 \mathrm{mmol} / \mathrm{L}$ has a better sensitivity and acceptable specificity in screening for diabetes and pre-diabetes in Sri Lankan adults.

Keywords: Fasting plasma glucose, Diabetes, pre-diabetes, Sri Lanka, South Asia

\section{Background}

Diabetes mellitus is a growing health concern recognized as a pandemic by the American Diabetes Association (ADA) and has its $80 \%$ of affected population from the developing countries. For adults aged $\geq 20$ years Sri Lanka has $10.3 \%(9.4-11.2 \%)$ overall prevalence of diabetes [males $9.8 \%(8.4-11.2 \%)$, females $10.9 \%$ (9.7$12.1 \%)]$ with a higher number of people with diabetes and pre-diabetes recorded form urban areas [1, 2]. But it is found that the number of people with diabetes identified in the rural areas of Sri Lanka has been progressively increasing with time [3] making diabetes no longer a disease of the rich.

\footnotetext{
* Correspondence: dissanayakeha@gmail.com

${ }^{2}$ Diabetes Research Unit, Department of Clinical Medicine, Faculty of

Medicine, University of Colombo, Colombo, Sri Lanka

Full list of author information is available at the end of the article
}

Fasting Plasma Glucose (FPG) of $\geq 7.0 \mathrm{mmol} / \mathrm{l}, 2$-h plasma glucose of $\geq 11.1 \mathrm{mmol} / \mathrm{L}$ after a standard $75 \mathrm{~g}$ Oral Glucose Tolerance Test (OGTT), HbA1c value of $\geq 6.5 \%$ and a casual blood glucose value of $\geq 11.1 \mathrm{mmol} /$ $\mathrm{L}$ in a patient with classic symptoms of hyperglycemia are the criteria recommended by the ADA for diagnosis of diabetes mellitus [4]. Many now tend to use HbA1c as a screening test for diagnosis as it does not require patients to fast and reflects longer-term glycaemia than plasma glucose [5-7]. But in resource limited settings the lack of standardization, relative unavailability and high cost of the HbA1c assay promote FPG and OGTT as screening tests for diabetes among physicians.

OGTT was considered as the gold standard test for diagnosis of diabetes by the World Health Organization. But it is inconvenient for the patient, cumbersome for laboratory personnel and reproducibility is poor. Therefore 
FPG is the popular diagnostic tool for diagnosis of diabetes and pre-diabetes among physicians. However OGTT remains the diagnostic tool for diagnosis of diabetes during pregnancy.

The ADA recommends FPG for the estimation of incidence and prevalence of diabetes. However FPG fails to detect a population with diabetes who will be detected using WHO 1999 criteria (OGTT $\geq 11.1 \mathrm{mmol} / \mathrm{L}$ ) [8, 9]. ADA defines pre-diabetes as either impaired fasting glucose (fasting plasma glucose between $5.6-6.9 \mathrm{mmol} /$ L) or impaired glucose tolerance (2-h OGTT glucose $7.8-11.0 \mathrm{mmol} / \mathrm{L}$ ).

The utility of OGTT and FPG in diagnosis of diabetes and pre-diabetes has not been assessed in Sri Lanka. OGTT was found to be more effective in diagnosing diabetes in American adults while FPG was found to be effective in those who are not willing to take OGTT [10]. The predictive ability of Impaired Fasting Glucose (IFG) on future diabetes risk was significantly low when compared to Impaired Glucose Tolerance (IGT) in some studies [11] while others have shown that future risk prediction of diabetes was similar for both IFG and IGT $[2,12]$.

There is a developing concern on modifying the cut-off values of FPG for diagnosing diabetes and pre-diabetes as it will increase the sensitivity for early detection [11, 13-15]. It was a necessity to develop cut off values specific to the region or country or to see the efficiency of ADA recommended cut-off values in our population as early detection and intervention will delay the progression of the disease.

\section{Methods}

Sri Lanka Diabetes and Cardiovascular Study (SLDCS) was a cross sectional study conducted by the Diabetes Research Unit of the Faculty of Medicine, University of Colombo and the Oxford Centre for Diabetes Endocrinology and Metabolism of the University of Oxford UK. Ethical approval was obtained from the Ethical Review Committee of the Faculty of medicine, University of Colombo, and all participants provided informed written consent. Data collection was conducted between August 2005 and September 2006.

\section{Study population}

SLDCS was carried out in seven out of all nine provinces in Sri Lanka; excluded were the North and the East provinces (14\% of the total population) due to security. The total sample frame was approximately 14 million people living in 12,018 'village officer' units. A multistage random cluster sampling technique was used to select a nationally representative sample of 5000 non-institutionalized adults $\geq 18$ years of age. The sample size of each province and the rural and urban populations of each province were determined using a probability proportional to the size technique. The sample was recruited from 100 clusters having 50 households in each. A cluster size was determined as 50 households in order to achieve a satisfactory spread of all groups of the population based on the prior experience of the investigators. Clusters were selected by a computer generated random number list from the smallest governmental community administrative unit the 'Village Office Units'. Voters' lists were used to randomly select the first household of each cluster and a uniform criterion was used to select the remaining 49 households in all 100 clusters. The selected households were visited by the study team to provide information of the study and to randomly select one eligible adult $\geq 18$ years from each household. Those who were pregnant, acutely ill or who declined participation were excluded.

\section{Data collection}

Temporary data collection centers were established within or in close proximity to each cluster. The selected subjects were advised to visit the centers in the morning between 7.30 a.m. and 9.00 a.m. after an overnight fast of 10-12 h. They were advised to follow an unrestricted diet and to continue with usual physical activities for at least 3 days prior to the date of data collection. A trained interviewer administered, structured and pre-coded questionnaire was used for data collection. All participants underwent OGTT. Blood for glucose estimation were collected into sodium fluoride/potassium oxalate tubes, centrifuged shortly after collection at the data collection centre and plasma was separated. Plasma and serum were stored in ice boxes until they were frozen at $-20^{\circ} \mathrm{C}$ within $6-12 \mathrm{~h}$ of collection. Biochemical tests were performed in a central laboratory in Colombo. Glucose assay was performed by an enzymatic (glucose oxidase) colorimetric method (Roche Diagnostics, Mannheim, Germany) in a Hitachi 704 chemical autoanalyser. The total coefficient of variation for glucose assay during the study period was $3.4 \%$.

\section{Data analysis}

Data was analysed using SPSS version17.0 (SPSS Inc. Released 2008. SPSS Statistics for Windows, Version 17.0. Chicago: SPSS Inc.). OGTT was considered as the reference standard for definition of diabetes and pre-diabetes, against which the FPG was compared to determine its diagnostic utility as a screening tool at population level.

\section{Results}

Among the total of 4485 participants, 4014 were included in the analysis after excluding people already on treatment for diabetes $(n=343)$ and those with missing data $(n=128)$. The mean age of the population was 45.3 $( \pm 15)$ years and $60.4 \%$ were females (Table 1 ). 
Table 1 Population characteristics

\begin{tabular}{ll}
\hline Parameter & Number/value \\
\hline Population analysed & 4014 \\
Age (years) (mean \pm SD) & $45.3( \pm 15.0)$ \\
Females (\%) & $60.4 \%$ \\
BMI $\left(\mathrm{kg} / \mathrm{m}^{2}\right)$ (mean $\left.\pm \mathrm{SD}\right)$ & $21.6( \pm 4.2)$ \\
FPG $(\mathrm{mmol} / \mathrm{L})($ mean \pm SD) & $4.85( \pm 1.09)$ \\
$2 \mathrm{~h}$ OGTT (mean \pm SD) & $6.14( \pm 2.86)$ \\
Newly diagnosed diabetes (\%) & $191(4.7)$ \\
Newly diagnosed IFG (\%) & $441(10.9)$ \\
Newly diagnosed IGT (\%) & $548(13.7)$
\end{tabular}

$B M I$ body mass index, FPG fasting plasma glucose, IFG impaired fasting glucose, IGT impaired glucose tolerance, OGTT oral glucose tolerance test

FPG between males $[5.10( \pm 1.02) \mathrm{mmol} / \mathrm{L}]$ and females $[5.06( \pm 1.08) \mathrm{mmo} / \mathrm{L}]$ did not show a significant difference but the $2 \mathrm{~h}$ post OGT glucose was higher in females $[6.39( \pm 2.81) \mathrm{mmol} / \mathrm{L}]$ compared to males [5.74 $( \pm 2.90) \mathrm{mmol} / \mathrm{L}](p<0.05)$ (Table 2). In both men and women FPG and post OGT plasma glucose increased significantly with age. Highest FPG was seen in 50-59 age group in both men and women. Highest post OGT plasma glucose was seen in $>70$ age group in both sexes. Men had significantly higher FPG compared to women in 20-29 and 30-39 age groups while women had significantly higher post OGT plasma glucose than men in $<19,20-29,30-39$ and 40-49 age groups (Table 2).

Area under the ROC curve was 0.76 for FPG in diagnosing diabetes while it was 0.91 for diagnosing pre-diabetes or diabetes (Fig. 1).

The sensitivity of FPG to diagnose pre-diabetes or diabetes is $43.8 \%$ when the cut off is $5.6 \mathrm{mmol} / \mathrm{L}$ with a specificity of $94.2 \%$ (Graph 1). The sensitivity to identify diabetes mellitus is $80.8 \%$ with a specificity of $92.1 \%$ at the same cut off value (Table 3). An FPG cut off value of 5.3 $\mathrm{mmol} / \mathrm{L}$ has noticeably higher sensitivity with reasonable specificity for diagnosis of pre-diabetes / diabetes (Table 3).

Table 2 FPG and OGTT plasma glucose in men and women across age groups $(N=4014)$

\begin{tabular}{|c|c|c|c|c|c|c|c|c|}
\hline \multirow{2}{*}{$\begin{array}{l}\text { Age } \\
\text { group }\end{array}$} & \multicolumn{4}{|c|}{$\mathrm{FPG}(\mathrm{mmol} / \mathrm{L})$} & \multicolumn{4}{|c|}{$2 \mathrm{~h}$ OGTT (mmol/L) } \\
\hline & $\overline{M e n}$ & Women & $P$ & $\overline{\text { Total }}$ & $\overline{M e n}$ & Women & $P$ & Total \\
\hline$\leq 19$ & 4.56 & 4.32 & 0.53 & 4.45 & 4.81 & 5.48 & 0.02 & 5.12 \\
\hline $20-29$ & 4.60 & 4.49 & 0.03 & 4.54 & 4.93 & 5.63 & $<0.01$ & 5.34 \\
\hline $30-39$ & 4.95 & 4.72 & 0.02 & 4.80 & 5.39 & 6.08 & $<0.01$ & 5.82 \\
\hline $40-49$ & 5.21 & 5.18 & 0.75 & 5.19 & 5.82 & 6.70 & $<0.01$ & 6.36 \\
\hline $50-59$ & 5.39 & 5.49 & 0.44 & 5.45 & 6.17 & 6.65 & 0.03 & 6.50 \\
\hline $60-69$ & 5.32 & 5.32 & 0.99 & 5.32 & 6.20 & 6.69 & 0.07 & 6.47 \\
\hline$\geq 70$ & 5.15 & 5.35 & 0.52 & 5.26 & 6.74 & 7.06 & 0.17 & 6.88 \\
\hline Total & 5.10 & 5.06 & 0.51 & 5.08 & 5.74 & 6.39 & $<0.01$ & 6.14 \\
\hline
\end{tabular}

$2 \mathrm{~h}$ OGTT 2-h oral glucose tolerance test, FPG fasting plasma glucose

\section{Discussion}

SLDCS is the largest population based study on diabetes conducted in Sri Lanka.

\section{Patterns of FPG and OGTT results}

Our female population had a higher overall post OGTT plasma glucose levels compared to males $(p<0.05)$. Relatively poor glucose handling was also seen in women in several other studies [16-20] but all involved women only in the middle age to elderly. Reason for this observation is uncertain. Longer gut half-life of glucose in women and inverse relationship between glucose tolerance and body height may at least partly explain this observation [16, 19]. Higher rates of IGT among women and rising OGT glucose values with age may contribute to higher risk of macro and microvascular complications of diabetes in this population. Currently practiced $2 \mathrm{~h}$ OGTT values for diagnosis of pre-diabetes and diabetes showed good sensitivity and specificity.

FPG was higher among men compared to women although the difference was statistically insignificant. Similar observations have been made in other studies [16-20]. Though the FPG reached a peak at 50-59 years, OGTT increased with aging. In contrast, studies in other countries have shown a significant increase in both FPG and post OGTT plasma glucose levels with aging [21-23] which is thought to be occurring partly due to reduced muscle mass and physical inactivity. This raises the need for the addition of post OGTT plasma glucose level for increasing the sensitivity for detection of diabetes in the elderly in our population.

\section{FPG cut off for pre-diabetes/diabetes screening}

According to our analysis, an FPG cut off value of 5.6 $\mathrm{mmol} / \mathrm{L}$ had a good specificity $(94.2 \%)$ for diagnosis of abnormal hyperglycemia (pre-diabetes and/or diabetes) but had a relatively poor sensitivity (43.8\%). But for diagnosis of diabetes alone it had higher sensitivity $(80.8 \%)$ and specificity $(92.1 \%)$. Once the cut off value was lowered furthermore it improved the sensitivity at the expense of minor lowering of specificity. We identified an FPG level of 5.3 $\mathrm{mmol} / \mathrm{L}$ as the most appropriate cut off value for our population in diagnosing both pre-diabetes and/or diabetes.

Lower cut off values for FPG were also recommended by other Asian countries ranging from $5.6-6.3 \mathrm{mmol} / \mathrm{L}$ for diagnosis of diabetes [13-15, 24]. However in contrast to our results for diagnosis of pre-diabetes, several other studies recommend a cut off value of $5.6 \mathrm{mmol} /$ $\mathrm{L}$ as a good cut off with a sensitivity and specificity close to $100 \%[25,26]$. Nevertheless none of the above studies involved South Asian population and in the majority participants were $>35$ years of age. What caused these differences in FPG levels in the Sri Lankan population is unclear. 


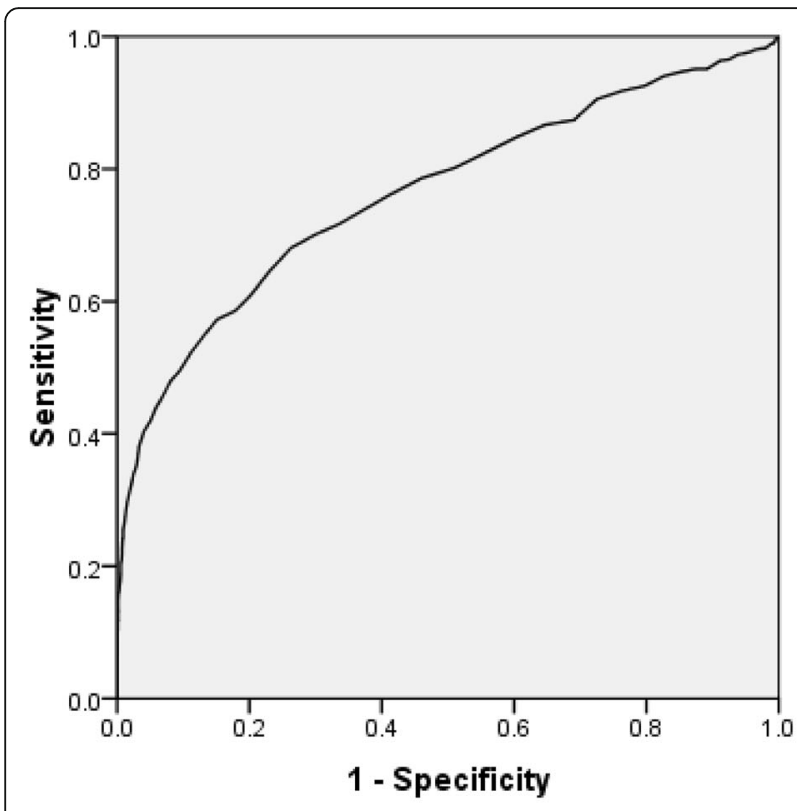

(a)

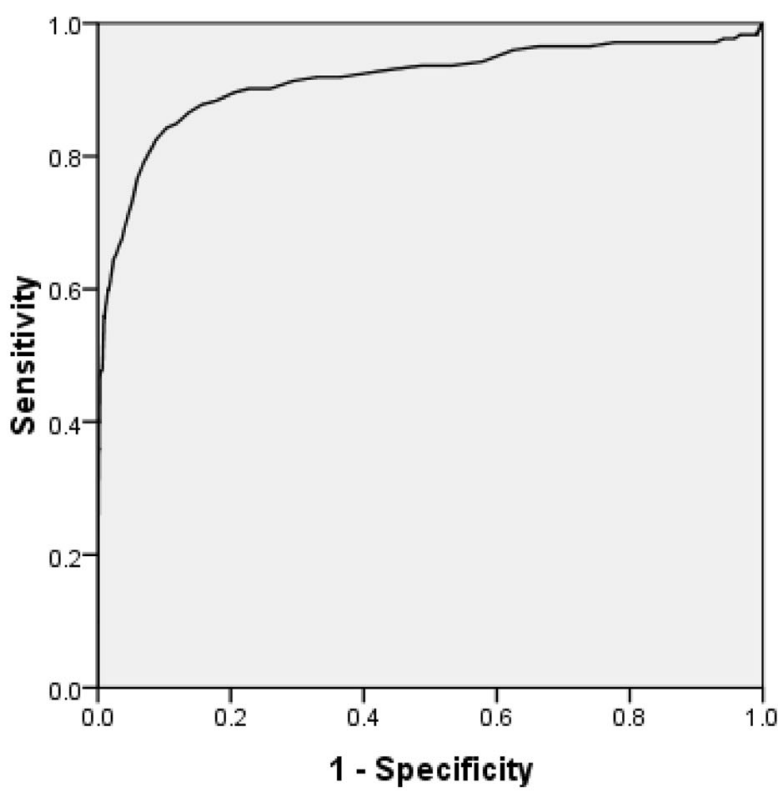

(b)

Fig. 1 ROC curve for FPG in screening (a) for pre-diabetes/diabetes; (b) for diabetes

We had several limitations in our study. First, only a single FPG or OGTT was used for defining the cases in our study as pre-diabetes or diabetes and a repeated confirmation test was not carried out. But we assume the impact of this to be very small due to the large number of participants. Second, the study did not include participants from North and East provinces. Data from more nationally representative sample as well as from other countries of the region will give more robust information about optimum cut points. Third, our study sample comprised of more women than men. However, no significant difference was observed in cut points for men and women (data not shown).

\section{Conclusions}

In resource limited settings, FPG and OGTT are valuable tests in diagnosing diabetes and pre- diabetes. However,

Table 3 Sensitivity and specificity for FPG in diagnosing both pre-diabetes/diabetes and diabetes

\begin{tabular}{llllll}
\hline FPG $(\mathrm{mmol} / \mathrm{L})$ & \multicolumn{2}{l}{ Pre DM and DM } & & DM only \\
\cline { 2 - 3 } FPG cut off & Sensitivity & Specificity & & Sensitivity & Specificity \\
\hline 4.4 & 87.4 & 30.9 & & 96.5 & 29.5 \\
5.0 & 68.1 & 73.7 & & 91.3 & 70.7 \\
5.3 & 54.7 & 87.0 & & 87.8 & 84.4 \\
5.6 & 43.8 & 94.2 & & 80.8 & 92.1 \\
6.1 & 26.6 & 98.9 & & 62.2 & 98.0 \\
7.0 & 15.8 & 99.8 & & 47.1 & 99.6 \\
\hline
\end{tabular}

ethnic and genetic variations in metabolism would suggest that single cut off may not be applicable to all universally. Fasting plasma glucose cut off of $5.3 \mathrm{mmol} / \mathrm{L}$ has a better sensitivity and acceptable specificity for diagnosis of pre-diabetes and diabetes among Sri Lankan adults. This will allow early identification of individuals at risk of progressing to diabetes and early targeted life style interventions to prevent development of diabetes and its complications. In addition, Sri Lankan female population has a relatively poor glucose handling compared to males and is more prominent in the reproductive age group. OGT also deteriorates with age than FPG. Therefore we suggest that performing OGTT in elderly would improve diagnostic rates of diabetes/pre-diabetes.

\section{Abbreviations}

ADA: American Diabetes Association; FPG: Fasting Plasma Glucose; HbA1c: Glycated haemoglobin A1; IFG: Impaired fasting glycaemia; IGT: Impaired glucose tolerance; OGT: Oral glucose tolerance; OGTT: Oral glucose tolerance test; SLDCS: Sri Lanka Diabetes and Cardiovascular Study; WHO: World Health Organization

\section{Acknowledgements}

The authors thank Diabetes Association of Sri Lanka and the World Health Organization office in Colombo and all individuals and institutions who helped and worked for the SLDCS.

\section{Funding}

SLDCS was funded by the National Science Foundation of Sri Lanka. Support provided by the Oxford Centre for Diabetes Endocrinology and Metabolism, UK, and the NIHR Biomedical Research Centre Programme is gratefully acknowledged. 


\section{Availability of data and materials}

Data set of the study is available with GWK and can be provided upon written request.

\section{Authors' contributions}

PK, MHRS, DRM and GWK developed the research protocol and supervised to study. GWK and SW supervised the conduct and quality control of laboratory analyses. HAD, CD and GWK analyzed the data. CD and HAD drafted the manuscript. GWK, PK, MHRS, SW and DRM critically reviewed the manuscript. All authors approved the final manuscript.

\section{Ethics approval and consent to participate}

All participants gave informed written consent for participation and dissemination of data. Ethical clearance was obtained from the Ethics Review Committee, Faculty of Medicine, University of Colombo, Sri Lanka.

\section{Consent for publication}

Participants provided consent for publication of data maintaining anonymity.

\section{Competing interests}

The authors declare that they have no competing interests.

\section{Publisher's Note}

Springer Nature remains neutral with regard to jurisdictional claims in published maps and institutional affiliations.

\section{Author details}

'Medical Research Institute, Colombo 08, Sri Lanka. ${ }^{2}$ Diabetes Research Unit, Department of Clinical Medicine, Faculty of Medicine, University of Colombo, Colombo, Sri Lanka. ${ }^{3}$ Cruddas Link Fellow, Harris Manchester University, University of Oxford, Oxford, UK. ${ }^{4}$ Oxford Centre for Diabetes Endocrinology and Metabolism, Oxford, London, UK. ${ }^{5}$ Faculty of Medicine, University of Colombo, Colombo, Sri Lanka.

Received: 16 November 2018 Accepted: 15 January 2019 Published online: 22 January 2019

\section{References}

1. Katulanda P, et al. Prevalence and projections of diabetes and pre-diabetes in adults in Sri Lanka--Sri Lanka diabetes, cardiovascular study (SLDCS). Diabet Med. 2008;25(9):1062-9.

2. McNeely MJ, et al. Comparison of a clinical model, the oral glucose tolerance test, and fasting glucose for prediction of type 2 diabetes risk in Japanese Americans. Diabetes Care. 2003;26(3):758-63.

3. Illangasekera U, Rambodagalla S, Tennakoon S. Temporal trends in the prevalence of diabetes mellitus in a rural community in Sri Lanka. J R Soc Promot Heal. 2004;124(2):92-4.

4. Standards of Medical Care in Diabetes-2012. Diabetes Care, 2012 35(Supplement 1): p. S11-S63.

5. Barr RG, et al. Tests of Glycemia for the diagnosis of type 2 diabetes mellitus. Ann Intern Med. 2002;137(4):263-72.

6. Saudek CD, et al. A new look at screening and diagnosing diabetes mellitus. J Clin Endocrinol Metab. 2008;93(7):2447-53.

7. Gillett MJ. International expert committee report on the role of the A1C assay in the diagnosis of diabetes. Diabetes Care. 2009;32(7):1327-34 Clin Biochem Rev, 2009. 30(4): p. 197-200.

8. Report of the Expert Committee on the Diagnosis and Classification of Diabetes Mellitus. Diabetes Care. 2002;25(suppl 1):s5-s20.

9. Harris Ml, et al. Comparison of diabetes diagnostic categories in the U.S. population according to the 1997 American Diabetes Association and 19801985 World Health Organization diagnostic criteria. Diabetes Care. 1997; 20(12):1859-62.

10. Zhang $P$, et al. Costs of screening for pre-diabetes among U.S. adults: a comparison of different screening strategies. Diabetes Care. 2003;26(9): 2536-42.

11. Gabir MM, et al. The 1997 American Diabetes Association and 1999 World Health Organization criteria for hyperglycemia in the diagnosis and prediction of diabetes. Diabetes Care. 2000;23(8):1108-12.

12. de Vegt F, et al. Relation of impaired fasting and postload glucose with incident type 2 diabetes in a Dutch population: the Hoorn study. Jama. 2001;285(16):2109-13.
13. Doi $Y$, et al. Fasting plasma glucose cutoff for diagnosis of diabetes in a Japanese population. J Clin Endocrinol Metab. 2008:93(9):3425-9.

14. Kim DJ, et al. Fasting plasma glucose cutoff value for the prediction of future diabetes development: a study of middle-aged Koreans in a health promotion center. J Korean Med Sci. 2005;20(4):562-5.

15. Mukai $\mathrm{N}$, et al. Cut-off values of fasting and post-load plasma glucose and $\mathrm{HbA1c}$ for predicting type 2 diabetes in community-dwelling Japanese subjects: the Hisayama study. Diabet Med. 2012;29(1):99-106.

16. Janghorbani M, Amini M. Effects of gender and height on the oral glucose tolerance test: the Isfahan diabetes prevention study. Rev Diabet Stud. 2008; 5(3):163-70.

17. Williams JW, et al. Gender differences in the prevalence of impaired fasting glycaemia and impaired glucose tolerance in Mauritius. Does sex matter? Diabet Med. 2003;20(11):915-20.

18. Hutchinson MS, et al. Effects of age and sex on estimated diabetes prevalence using different diagnostic criteria: the Troms\&\#xf; OGTT study. Int J Endocrinol. 2013;2013:9.

19. Anderwald C, et al. Mechanism and effects of glucose absorption during an oral glucose tolerance test among females and males. J Clin Endocrinol Metab. 2011;96(2):515-24.

20. Faerch $\mathrm{K}$, et al. Sex differences in glucose levels: a consequence of physiology or methodological convenience? The Inter99 study. Diabetologia. 2010;53(5):858-65.

21. Ko GT, Wai HP, Tang JS. Effects of age on plasma glucose levels in nondiabetic Hong Kong Chinese. Croat Med J. 2006;47(5):709-13.

22. Toyoda $\mathrm{K}$, et al. Factors responsible for age-related elevation in fasting plasma glucose: a cross-sectional study in Japanese men. Metabolism. 2008; 57(2):299-303.

23. Bando $Y$, et al. The relationship of fasting plasma glucose values and other variables to 2-h Postload plasma glucose in Japanese subjects. Diabetes Care. 2001;24(7):1156-60.

24. Cockram CS, et al. Assessment of glucose tolerance test criteria for diagnosis of diabetes in Chinese subjects. Diabetes Care. 1992;15(8):988-90.

25. Guerrero-Romero F, Rodriguez-Moran M. Lowered criterion for normal fasting plasma glucose: impact on the detection of impaired glucose tolerance and metabolic syndrome. Arch Med Res. 2006;37(1):140-4.

26. Zhang $P$, et al. Efficient cutoff points for three screening tests for detecting undiagnosed diabetes and pre-diabetes: an economic analysis. Diabetes Care. 2005;28(6):1321-5.

Ready to submit your research? Choose BMC and benefit from:

- fast, convenient online submission

- thorough peer review by experienced researchers in your field

- rapid publication on acceptance

- support for research data, including large and complex data types

- gold Open Access which fosters wider collaboration and increased citations

- maximum visibility for your research: over $100 \mathrm{M}$ website views per year

At $\mathrm{BMC}$, research is always in progress.

Learn more biomedcentral.com/submissions 